\title{
Comparison of Contemporary Evolutionary Algorithms on the Rotated Klee-Minty Problem
}

\author{
Supplementary Material
}

This supplementary material accompanies the GECCO 2019 workshop paper of the same title and the same authors. It contains additional experimental data in tabular form. In particular, the quality indicators illustrated in Fig. 3 and Fig. 4 of the workshop paper are provided.

The indicators aggregate the results obtained from 15 independent algorithm runs by making use of the lexicographic ordering relation $\preceq_{\text {lex }}$, refer to Eq. (4). The following indicators are displayed together with the optimal fitness value $f_{\text {opt }}=N^{3}$ for each search space dimension considered $(N \in$ $\{2,3,5,10,20,40\})$. For a more detailed examination of the mutual algorithm performance, these significant differences among these indicators can also be examined by use of statistical hypothesis testing, e.g. by using the Wilcoxon singed-rank test.

\begin{tabular}{cl}
\hline$f_{\text {best }}$ & fitness of the best solution found in all 15 runs \\
$f_{\text {med }}$ & fitness of the median solution \\
$\nu_{\text {med }}$ & constraint violation of the median solution \\
$\left|f_{\text {med }}-f_{\text {opt }}\right|$ & $\begin{array}{l}\text { absolute fitness error of the median solution } \\
\text { feasibility rate } F R \text { defined as the quotient of those runs that re- } \\
\text { alized a feasible solution and the total number of algorithm runs }\end{array}$ \\
$\left\|\mathbf{y}-\mathbf{y}_{\text {opt }}\right\|$ & $\begin{array}{l}\text { mean deviation of all final and feasible algorithm realizations } \mathbf{y} \\
\text { from the known optimal solution } \mathbf{y}^{*} \text { over } 15 \text { algorithm runs }\end{array}$ \\
meanFevals & mean number of function evaluations consumed until termination \\
\hline
\end{tabular}

The final algorithm results corresponding to the comparison of eight algorithms considered in Sec. 4.1 of the workshop paper are presented in Tables 1 to 8 . The presentation style is inspired by the CEC competitions on constrained real-parameter optimization. It allows for comparing different algorithms with respect to realized median objective function values. Further, algorithm performance in the search space is measured by taking into account the mean deviation of the best found candidate solution from the optimal solution. An illustration of these quality indicators is given in Fig. 3 and 4 of the workshop paper (provided that the respective algorithm realizes feasible solutions in each of the 15 runs).

The results displayed in Tables 9 to 11 correspond to the algorithm variants that replace the $\epsilon$-level constraint handling with the lexicographic ordering approach. These algorithms are taken into account within Sec. 4.2 (Fig. 5). Table 12 provides the final results of the iUDE variant that establishes a fast reduction of the $\epsilon$-threshold. This algorithm variant is considered in Fig. 6 of the workshop paper. 


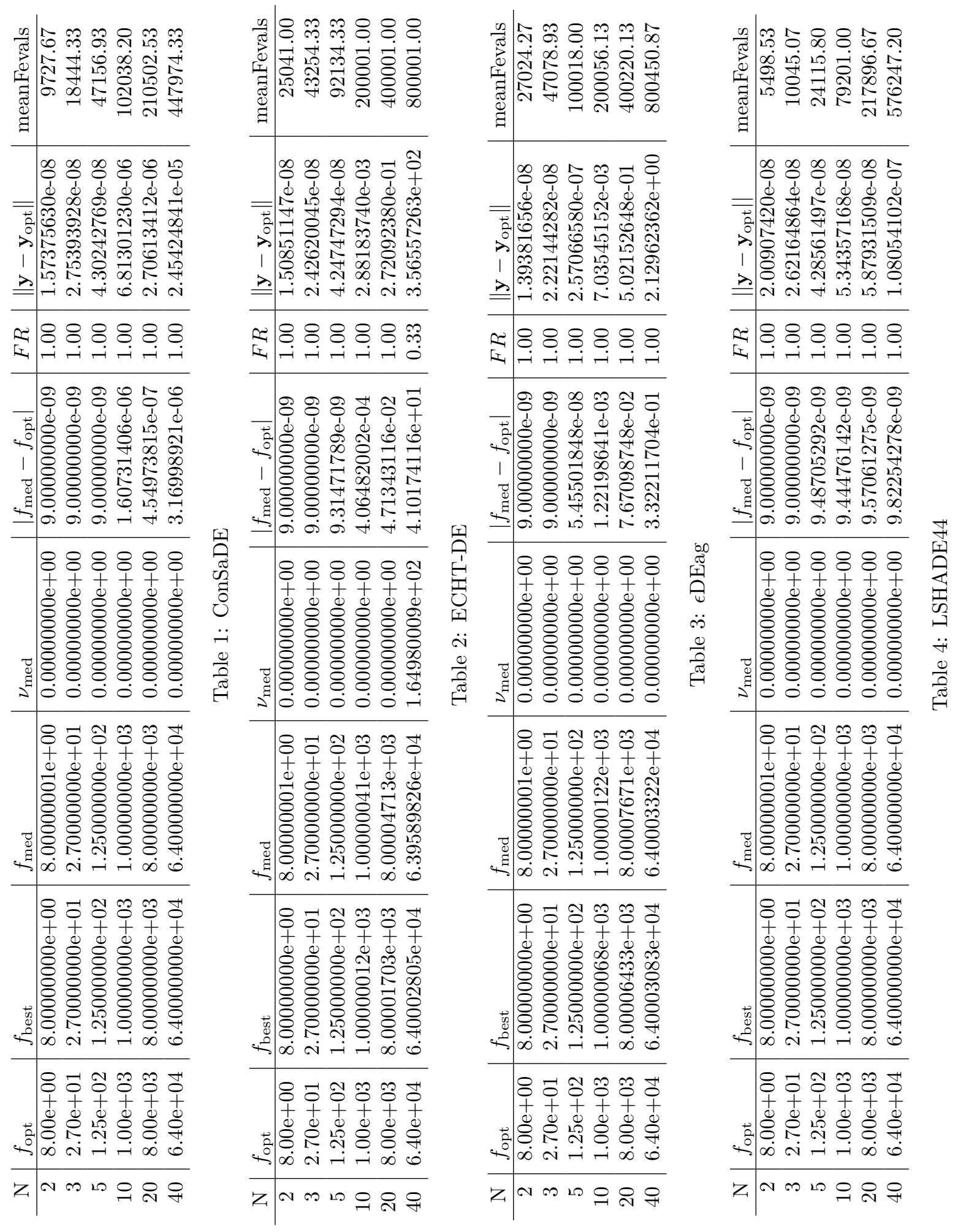



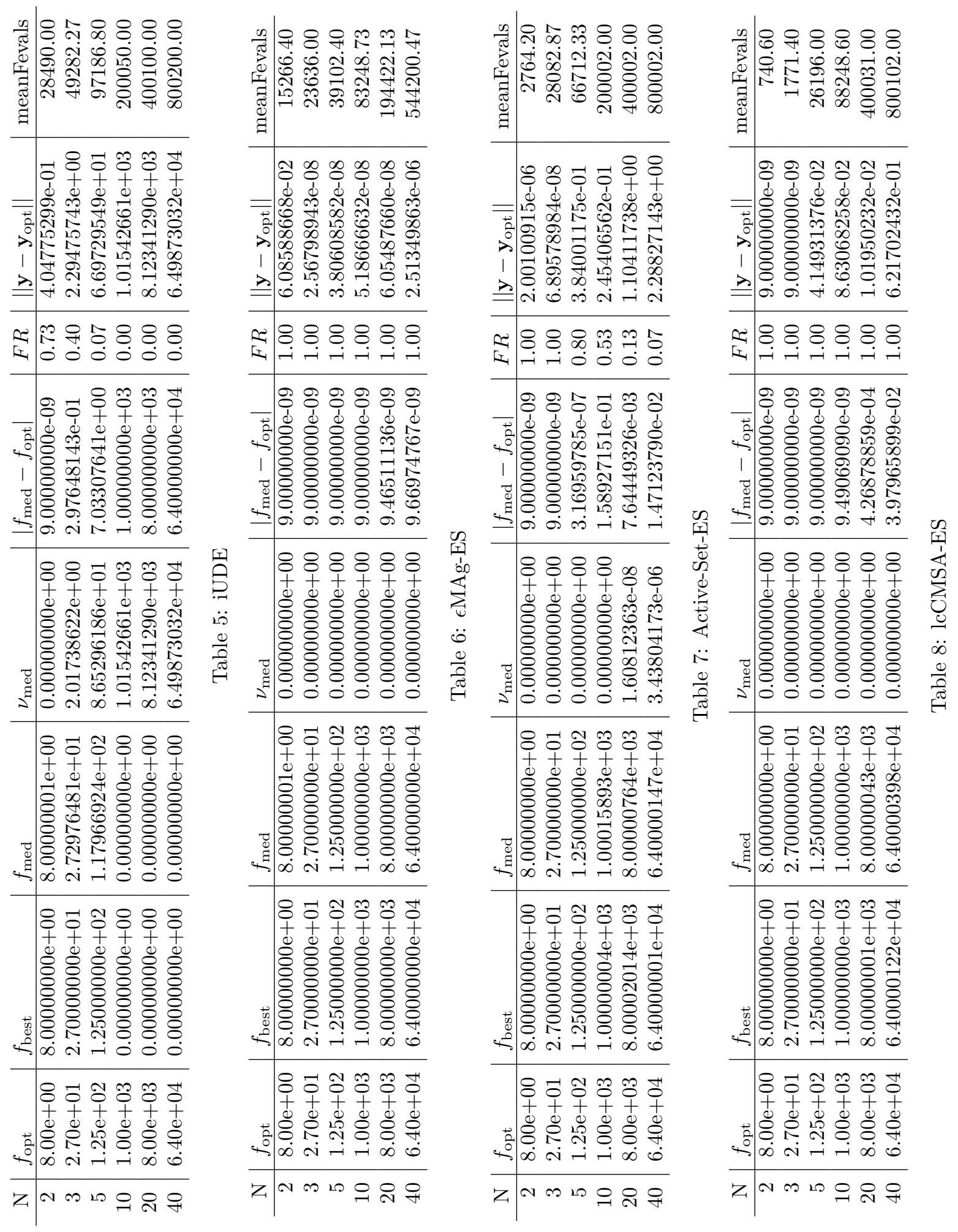


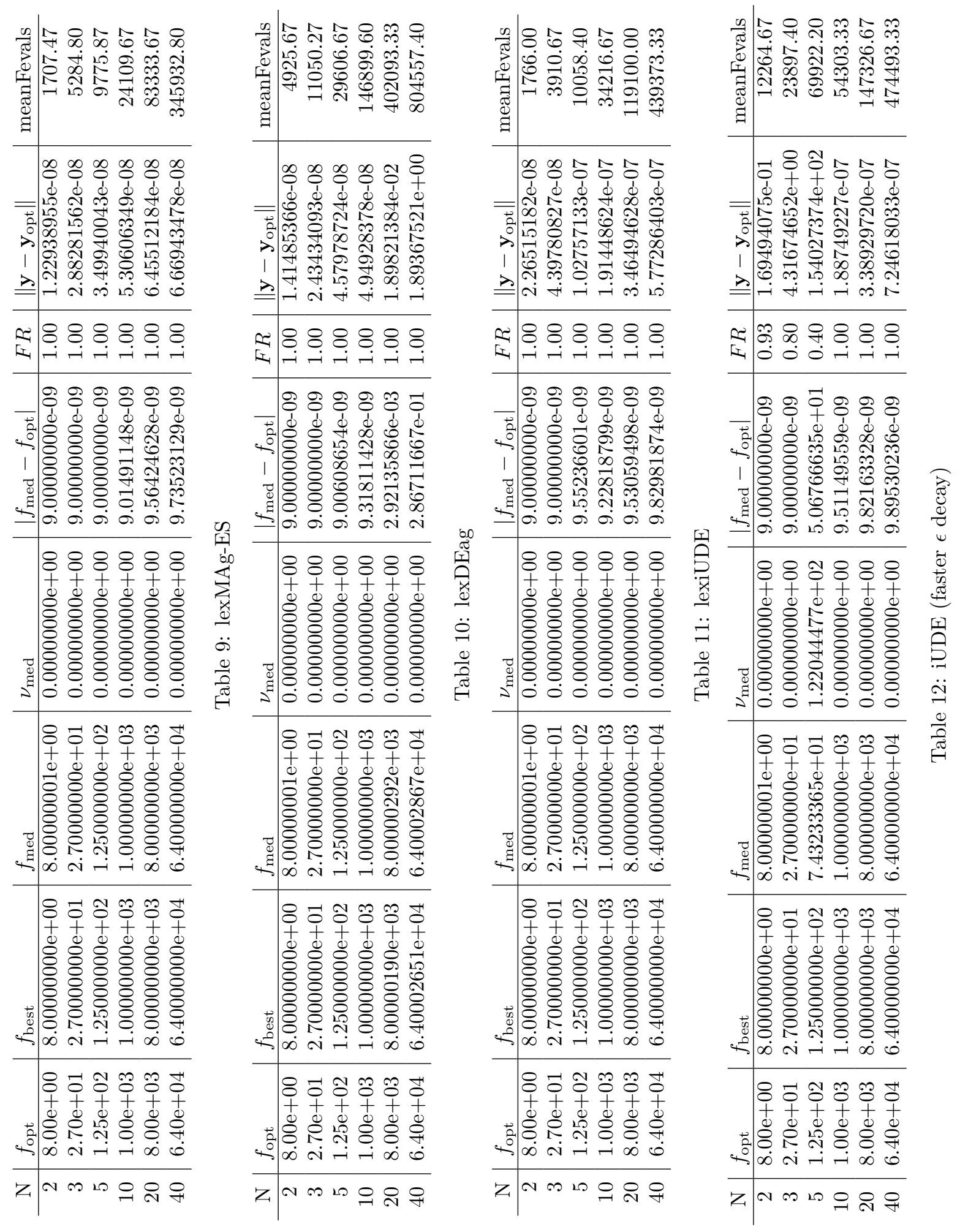

